\title{
Nine Formulations of Quantum Mechanics
}

\author{
Daniel F. Styer, Miranda S. Balkin, Kathryn M. Becker, \\ Matthew R. Burns, Christopher E. Dudley, Scott T. Forth, \\ Jeremy S. Gaumer, Mark A. Kramer, David C. Oertel, Leonard H. Park, \\ Marie T. Rinkoski, Clait T. Smith, and Timothy D. Wotherspoon \\ Department of Physics \\ Oberlin College \\ Oberlin, Ohio 44074 \\ Dan.Styer@oberlin.edu \\ American Journal of Physics \\ 70 (3), March 2002, 288-297
}


1 The matrix formulation (Heisenberg)

$$
\frac{d \hat{A}(t)}{d t}=-\frac{i}{\hbar}[\hat{A}(t), \hat{H}]
$$




\section{The wavefunction formulation (Schrödinger)}

For a two-particle non-relativistic system neglecting spin,

$$
\frac{\partial \psi\left(\mathbf{x}_{1}, \mathbf{x}_{2}, t\right)}{\partial t}=-\frac{i}{\hbar}\left[-\frac{\hbar^{2}}{2 m_{1}} \nabla_{1}^{2} \psi\left(\mathbf{x}_{1}, \mathbf{x}_{2}, t\right)-\frac{\hbar^{2}}{2 m_{2}} \nabla_{2}^{2} \psi\left(\mathbf{x}_{1}, \mathbf{x}_{2}, t\right)+V\left(\mathbf{x}_{1}, \mathbf{x}_{2}\right) \psi\left(\mathbf{x}_{1}, \mathbf{x}_{2}, t\right)\right] .
$$




\section{The path integral formulation (Feynman)}

Suppose, for example, that a single particle is located at point $\mathbf{x}_{i}$ when the time is $t_{i}$, and we wish to find the probability that it will be located at $\mathbf{x}_{f}$ when the time is $t_{f}$. This probability is calculated as follows:

- Enumerate all classical paths from the initial to the final state.

- Calculate the classical action $S=\int$ (Lagrangian) $d t$ for each path.

- Assign each path a "transition amplitude" proportional to $e^{i S / \hbar}$. (The proportionality constant is adjusted to assure normalization.)

- Sum the amplitude over all paths. (Because there is a continuum of paths, this "sum" is actually a particular kind of integral called a "path integral.")

- The resulting sum is the transition amplitude, and its square magnitude is the transition probability.

For different problems - such as a particle changing from one momentum to another, or for an initial state that has neither a definite position nor a definite momentum — variations on this procedure apply. 


\section{Phase space formulation (Wigner)}

For a single particle restricted to one dimension, the Wigner phase-space distribution function is

$$
W(x, p, t)=\frac{1}{2 \pi \hbar} \int_{-\infty}^{+\infty} \psi^{*}\left(x-\frac{1}{2} y, t\right) \psi\left(x+\frac{1}{2} y, t\right) e^{-i p y / \hbar} d y
$$

It evolves in time as

$$
\frac{\partial W(x, p, t)}{\partial t}=-\frac{p}{m} \frac{\partial W(x, p, t)}{\partial x}-\int_{-\infty}^{+\infty} K\left(x, p^{\prime}\right) W\left(x, p+p^{\prime}, t\right) d p^{\prime}
$$

where the kernel $K(x, p)$ is

$$
K(x, p)=\frac{1}{2 \pi \hbar^{2}} \int_{-\infty}^{+\infty}\left[V\left(x-\frac{1}{2} y\right)-V\left(x+\frac{1}{2} y\right)\right] \sin (p y / \hbar) d y .
$$




\section{Density matrix formulation}

The density matrix corresponding to a pure state $|\psi\rangle$ is the outer product

$$
\hat{\rho}=|\psi\rangle\langle\psi|
$$

It evolves in time as

$$
\frac{d \hat{\rho}(t)}{d t}=+\frac{i}{\hbar}[\hat{\rho}(t), \hat{H}]
$$




\section{Second quantization formulation}

You know this one. 


\section{Variational formulation}

The central entity in this formulation remains the wavefunction $\psi\left(\mathbf{x}_{1}, \mathbf{x}_{2}, t\right)$, but the rule for time evolution is no longer the Schrödinger equation. Of all possible normalized wavefunctions $\psi\left(\mathbf{x}_{1}, \mathbf{x}_{2}, t\right)$, the correct wavefunction is the one that minimizes the "action integral" over time and configuration space, namely

$$
\int d t \int d^{3} x_{1} \int d^{3} x_{2} \mathcal{L}\left(\mathbf{x}_{1}, \mathbf{x}_{2}, t\right)
$$

where the "Lagrangian density" is

$$
\mathcal{L}\left(\mathbf{x}_{1}, \mathbf{x}_{2}, t\right)=\hbar \Im m\left\{\psi^{*} \frac{\partial \psi}{\partial t}\right\}+\frac{\hbar^{2}}{2 m_{1}} \nabla_{1} \psi^{*} \cdot \nabla_{1} \psi+\frac{\hbar^{2}}{2 m_{2}} \nabla_{2} \psi^{*} \cdot \nabla_{2} \psi+V\left(\mathbf{x}_{1}, \mathbf{x}_{2}\right) \psi^{*} \psi .
$$




\section{The pilot wave formulation (de Broglie-Bohm)}

For a two-particle system, there is a "pilot wave" in configuration space as well as two particles with definite positions in real space.

$$
\psi\left(\mathbf{x}_{1}, \mathbf{x}_{2}, t\right)=R\left(\mathbf{x}_{1}, \mathbf{x}_{2}, t\right) e^{i S\left(\mathbf{x}_{1}, \mathbf{x}_{2}, t\right) / \hbar}
$$

If one defines the state-dependent "quantum potential"

$$
Q\left(\mathbf{x}_{1}, \mathbf{x}_{2}, t\right)=-\frac{\hbar^{2}}{2 m_{1}} \frac{\nabla_{1}^{2} R}{R}-\frac{\hbar^{2}}{2 m_{2}} \frac{\nabla_{2}^{2} R}{R}
$$

then the pilot wave evolves in time according to

$$
\frac{\partial S}{\partial t}=-\frac{\left(\nabla_{1} S\right)^{2}}{2 m_{1}}-\frac{\left(\nabla_{2} S\right)^{2}}{2 m_{2}}-V\left(\mathbf{x}_{1}, \mathbf{x}_{2}\right)-Q\left(\mathbf{x}_{1}, \mathbf{x}_{2}, t\right)
$$

and

$$
\frac{\partial P}{\partial t}+\frac{1}{m_{1}} \nabla_{1} \cdot\left(P \nabla_{1} S\right)+\frac{1}{m_{2}} \nabla_{2} \cdot\left(P \nabla_{2} S\right)=0
$$

where

$$
P\left(\mathbf{x}_{1}, \mathbf{x}_{2}, t\right)=R^{2}\left(\mathbf{x}_{1}, \mathbf{x}_{2}, t\right) .
$$

The two point particles move with accelerations

$$
m_{1} \frac{d \mathbf{v}_{1}}{d t}=-\nabla_{1} V-\nabla_{1} Q \quad \text { and } \quad m_{2} \frac{d \mathbf{v}_{2}}{d t}=-\nabla_{2} V-\nabla_{2} Q
$$




\section{The Hamilton-Jacobi formulation}

$$
\begin{gathered}
\psi\left(\mathbf{x}_{1}, \mathbf{x}_{2}, t\right)=\exp \left[i S\left(\mathbf{x}_{1}, \mathbf{x}_{2}, t\right) / \hbar\right] \\
\frac{\partial S}{\partial t}=i \frac{\hbar}{2 m_{1}} \nabla_{1}^{2} S-\frac{1}{2 m_{1}} \nabla_{1} S \cdot \nabla_{1} S+i \frac{\hbar}{2 m_{2}} \nabla_{2}^{2} S-\frac{1}{2 m_{2}} \nabla_{2} S \cdot \nabla_{2} S-V\left(\mathbf{x}_{1}, \mathbf{x}_{2}\right) .
\end{gathered}
$$




\section{Summary and conclusions}

We have discussed nine distinct formulations of quantum mechanics. Did we learn anything in the process? The most profound lesson is already familiar from classical mechanics, and indeed from everyday life: "There is no sliver bullet." Each of these formulations can make some application easier or some facet of the theory more lucid, but no formulation produces a "royal road to quantum mechanics." Quantum mechanics appears strange to our classical eyes, so we employ mathematics as our sure guide when intuition fails us. The various formulations of quantum mechanics can repackage that strangeness, but they cannot eliminate it.

The matrix formulation, the first formulation to be discovered, is useful in solving harmonic oscillator and angular momentum problems, but for other problems it is quite difficult. The ever-popular wavefunction formulation is standard for problem solving, but leaves the conceptual misimpression that wavefunction is a physical entity rather than a mathematical tool. The path integral formulation is physically appealing and generalizes readily beyond the domain of non-relativistic quantum mechanics, but is laborious in most standard applications. The phase space formulation is useful in considering the classical limit. The density matrix formulation can treat mixed states with ease, so it is of special value in statistical mechanics. The same is true of second quantization, which is particularly important when large numbers of identical particles are present. The variational formulation is rarely the best tool for applications, but it is valuable in extending quantum mechanics to unexplored domains. The pilot wave formulation brings certain conceptual issues to the fore. And the Hamilton-Jacobi formulation holds promise for solving otherwise-intractable bound state problems.

We are fortunate indeed to live in a universe where nature provides such bounty. 